\title{
Emergence of growth and dormancy from a kinetic model of the Escherichia coli central carbon metabolism
}

\section{Yusuke Himeoka}

University of Tokyo

Namiko Mitarai ( $D$ mitarai@nbi.ku.dk)

University of Copenhagen https://orcid.org/0000-0003-0116-7606

\section{Article}

Keywords:

Posted Date: January 7th, 2022

DOI: https://doi.org/10.21203/rs.3.rs-1193900/v1

License: (c) (1) This work is licensed under a Creative Commons Attribution 4.0 International License.

Read Full License 


\title{
Emergence of growth and dormancy from a kinetic model of the Escherichia coli central carbon metabolism
}

\author{
Yusuke Himeoka*, Namiko Mitarai ${ }^{\dagger}$
}

\begin{abstract}
Physiological states of bacterial cells exhibit a wide spectrum of timescale. Under nutrient-rich conditions, most of the cells in an isogenic bacterial population grow at certain rates, while a small subpopulation sometimes stays in a dormant state where the growth rates slow down by orders of magnitude. For revealing the origins of such heterogeneity of timescales, we studied the kinetic model of Escherichia coli central carbon metabolism including the dynamics of the energy currency molecules. We found that the model robustly exhibits both the growing- and the dormant state. In order to unveil the mechanism of distinct behaviours, we developed a recursive method to simplify the model without changing the qualitative feature of the dynamics. Analytical and numerical studies of the 2-variable minimal model revealed the necessary conditions for the distinct behaviour, namely, the depletion of energy due to the futile cycle and its non-uniform impact on the kinetics because of the coexistence of the energy currency-coupled and uncoupled reactions as well as branching of the network. The result is consistent with the experimental reports that the dormant cells commonly exhibit low ATP levels, and provides a possible explanation for the appearance of dormant cells that causes antibiotic persistence.
\end{abstract}

Bacterial growth rates span in a wide range of timescales: Escherichia coli cells typically double every 20 minutes under the nutrient-rich conditions, while cells can also exhibit dormancy where the growth of cells almost halts and yet the death is strongly suppressed [1-3]. The transition to the dormant states can either be a stochastic event or a response to hostile environments such as starvation and exposure to antibiotics. This dormancy is a beneficial strategy for surviving the nutrient-poor conditions as it can lower the cell's nutrient requirements [4]. Also, the dormancy is known as the main cause of bacterial persistence that has a high tolerance to antibiotics, and thus, has been gathering attention from a wide range of fields from microbiology to therapeutic studies $[2,3,5,6]$.

Notable changes in the timescale of cellular physiology are happening in the dormant cells. It has been implied that the dormant cells have a sort of memory capacities: The lag time was shown to depend on the length of time that the cells are starved $[7,8]$ and the death rates of the starved cells differ depending on the previous culture conditions even though the starvation condition is identical [9]. Given that slow dynamics are vital for storing memories, a drastic change in the timescale of cellular physiology is necessary. Indeed, it was reported that the proteome kept changing at least for 8 hours in the starved E.coli cells [10].

How does the transition to the dormancy happen? Experimental studies of E.coli have revealed the links between the dormancy and several molecules. Increased alarmone (p)ppGpp modulates bacterial physiology by transcriptional reprogramming and the direct adjustments of the target proteins $[11,12]$. One of the alternative sigma factors $\sigma^{S}$ activates a number of stress response genes [13,14]. Toxin-antitoxin modules lead to the growth arrest by inhibiting essential cellular processes, such as DNA replication and protein translation [15].

In contrast to such a "genetically-controlled" view of bacterial dormancy, Kim Lewis and colleagues proposed a different, "low-energy" perspective: Decreases in ATP concentration itself lead to dormancy and persistence. The single-cell level ATP quantification revealed that the cells that survived the ampicillin treatment had significantly lower ATP concentrations prior to the antibiotic exposure [16]. A similar scenario

*Universal Biology Institute, University of Tokyo, 7-3-1 Hongo, Bunkyo-ku, Tokyo, Japan

${ }^{\dagger}$ The Niels Bohr Institute, University of Copenhagen, Blegdamsvej 17, Copenhagen, 2100, Denmark 
was proposed by the group also for the pathogenic bacterium, Staphylococcus aureus. They added a sublethal amount of arsenate which decreases cellular ATP concentrations [17] to the culture ahead of the antibiotic treatment. Then, the survival fraction of the cells from the ciprofloxacin exposure considerably increased [18].

Motivated by this low-energy hypothesis $[16,18]$ and studies reporting other roles of ATP depletion in dormancy $[19,20]$, we analyze a link between the metabolic dynamics and the growth-dormancy transition using a mathematical model. Here, we note that there is a clear time-scale separation between the metabolic dynamics $(\approx$ seconds [21]) and the modulation of protein levels $(\approx$ hours [22]). Thus, the kinetic model of metabolism without explicit genetic regulations should capture the characteristic nature of the transition if the metabolic dynamics, by itself, triggers the growth-dormancy transition.

Therefore, in the present manuscript, we study the kinetic model of E.coli central carbon metabolism with the cofactors such as ATP included as variables. To the best of our knowledge, this is the first kinetic model of E.coli metabolism where the dynamics of cofactors are dealt as variables: There are a number of studies of kinetic model of E.coli central carbon metabolism [23-38]. However, as far as we know, the dynamics of the cofactors are neglected [23-33], or if included, the relaxation dynamics of the models are not actually computed [34-38]. As the experimental studies suggested $[16,18]$, ATPs may play a central role in the transition to the dormancy. Thus, the cofactors can be vital components of the model for studying the growth-dormancy transition.

In the following sections, we present that the kinetic model of E.coli central carbon metabolism with cofactors robustly exhibits two distinct dynamics: One is reminiscent of the normal growth behaviour and the other is analogous to the dormant dynamics. Then we derive the minimal network showing qualitatively the same dynamics. The analysis of the minimal model reveals two necessary conditions for the emergence of both growth and dormant dynamics, namely, the depletion of energy due to the futile cycle and its nonuniform impact on the kinetics because of the coexistence of the energy currency-coupled and uncoupled reactions as well as branching of the network.

The obtained result implies that the depletion of ATP and ADP itself leads to the slow dynamics of the metabolites' concentrations. This conclusion is consistent with the "low-energy" view of the bacterial dormancy presented in $[16,39]$, and highlights the notable impact of introducing cofactors into models. We also discuss the possible applications of our analysis for the studies of dormancy in other species, based on the minimal network motifs.

\section{Results}

\section{Model}

In the present manuscript, we study the E. coli core network [40] as one of the simplest models of the real metabolic reaction networks. The $E$. coli core model was obtained from BiGG database [41]. The model contains stoichiometry and the reversibility of the reactions. The E. coli core model has 52 and 75 intracellular metabolites and reactions, respectively. After an appropriate data curation as described later, we implemented the model by using the ordinary differential equation (ODE) that describes the dynamics of concentrations of metabolites.

We applied several modifications to the model to make it suitable for the ODE implementation. First, small molecules such as $\mathrm{O}_{2}, \mathrm{H}_{2} \mathrm{O}$, and $\mathrm{NH}_{4}$, were not considered as variables but treated as constants under the assumptions that the external concentration of these chemicals are kept constant and uptakes/secretions of them take place quickly. The uptake and secretion pathways of all carbon sources except glucose are removed.

Under the anaerobic conditions, cells transfer the free energy to ATP directly, while under the aerobic conditions, most of the energy transfer takes an indirect form: the energy is first transferred to other chemicals such as NADH and NADPH, and then, the stored energy in NADH, NADPH, and others are used for converting ADP to ATP. The conversion yield of ATP per NADH in the E. coli core model is $1.25^{1}$ and $\mathrm{NADH} / \mathrm{NADPH}$ yield is roughly unity. For introducing the cofactors to the model in a simple manner, we

\footnotetext{
${ }^{1}$ via NADH16, CYTB, and ATPS4r.
} 
assume that the energy transfers via $\mathrm{NADH}$ and $\mathrm{NADPH}$ are sufficiently fast and ATP/NADH(NAPDH) yield as unity. According to these assumptions, we replace NAD(NADP) and NADH(NADPH) with ADP and ATP, respectively (we discussed the validity of this assumption in the Discussion section and SI text Section.9). Full lists of the chemical components and the reactions are provided in SI Data.1.

Also, the stoichiometry of the growth reaction was modified. The original E. coli core model has the biomass production reaction leading to the cell growth consisting of 16 substrates and 7 products with noninteger stoichiometry constants. For the kinetic modeling, such reactions having too many substrates and products leads to numerical instability and non-integer stoichiometry is unreasonable. Thus, we replaced the biomass production reaction with a following reaction: (Erythrose 4-phosphate) + (L-Glutamine) + (ATP) $\rightarrow(\mathrm{ADP})$. This reaction is much simpler than the original one but requires the model to run all the modules of the metabolic reactions, namely pentose phosphate pathway for Erythrose 4-phosphate (e4p), TCA cycle for L-Glutamine $(g \ln )$, and energy generation for ATP. Hereafter, we call this simplified biomass production reaction as the growth reaction.

The resulting model consists of 32 variables and 40 reactions. The final metabolic reaction network is drawn in Fig. 1. Our model cell takes up the nutrient from the node labeled as "glc" which has a constant concentration, performs successive conversion of the chemicals with generating energy, and proceeds the growth reaction.

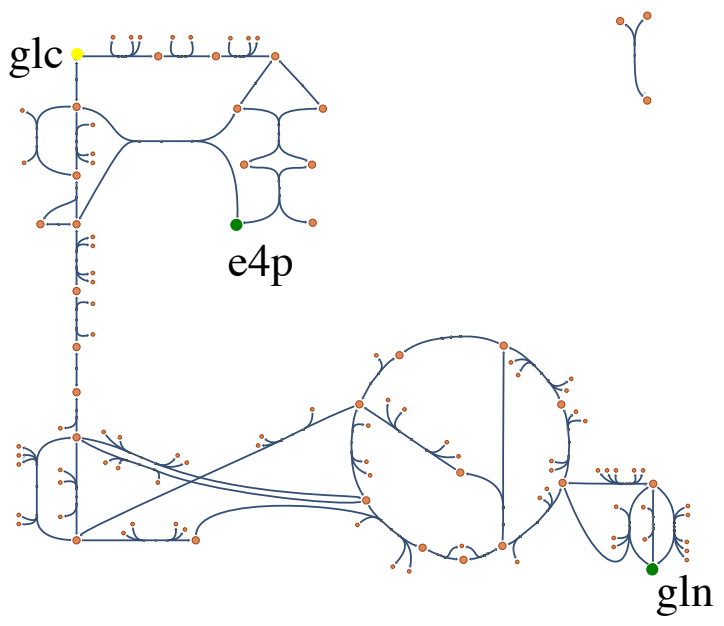

Figure 1: The metabolic network of the E. coli core model generated by Escher [41]. The sole carbon source (glucose-6-phosphate) is placed at the left top (abbreviated as glc). We highlighted the substrates of the growth reaction other than ATP, namely, e4p and gln. The growth reaction is not drawn.

First, we simulated the model with realistic setups. The kinetic parameters of $E$. coli core model have been estimated using the metabolic ensemble modeling (MEM) by Khodayari and colleagues [36]. We derived the Michaelis-Menten type rate equation for each reaction according to the enzyme kinetics used in [36] with the presented kinetic parameters. Then we assumed that each chemical species is consumed/synthesized by associated reactions, diluted as the cell grows, and spontaneously degraded at a slow rate. Thus, the temporal change of the concentration of the $i$ th chemical species $X_{i}$ is ruled by

$$
\frac{d\left[X_{i}\right]}{d t}=\sum_{j} S_{i j} J_{j}-d\left[X_{i}\right]-\mu\left[X_{i}\right],
$$

where $S$ is the stoichiometric matrix and $J_{i}$ 's are the fluxes due to chemical reactions. $d$ and $\mu$ are the spontaneous degradation rate and the growth rate, respectively. Note that the concentrations of enzymes are supposed to be constant and lumped in the kinetic parameters. We assumed that spontaneous degradation 
A

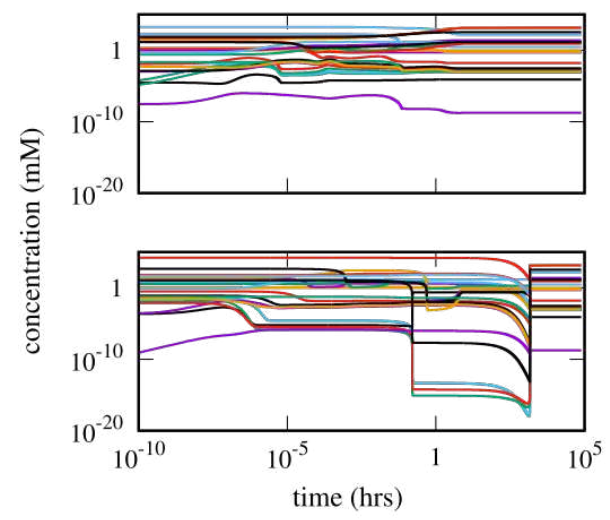

B

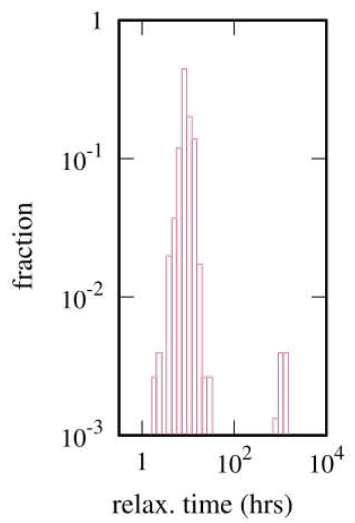

$\mathrm{C}$

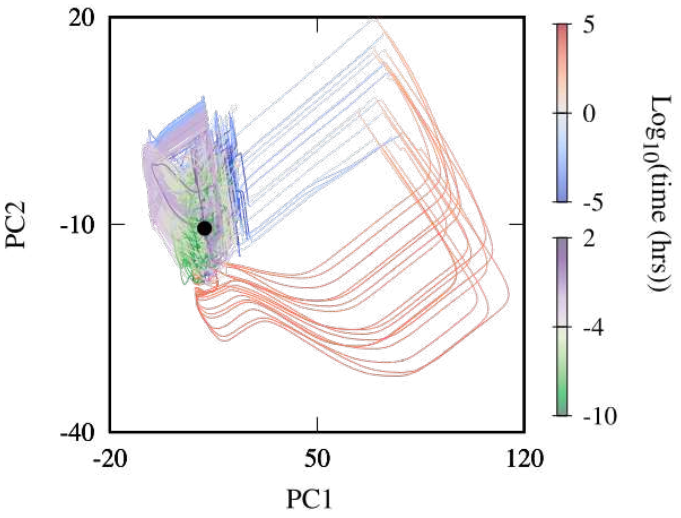

Figure 2: A. Two characteristic dynamics of E. coli core model starting from different initial points. While the growth rate of the cell is $\approx 0.5$ per hour at the attractor, there are huge differences in the relaxation behaviours between the top and bottom panel. B. The distribution of the relaxation time showing a clear bimodality. C. Trajectories are overlaid in 2-dimensional principal component space. Color indicates $\log _{10}$ of time. The trajectories having shorter relaxation time (several hours) are colored in green-white-purple while the others are colored in blue-white-red. The black point corresponds to the steady-state attractor. Initial concentration of each metabolites is $10^{u_{i, n}}\left[X_{i}^{(\mathrm{ss})}\right](\mathrm{mM})$ with $\left[X_{i}^{(\mathrm{ss})}\right]$ as the steady-state concentration of the $i$ th metabolite, and $\left.u_{i}^{(} n\right)$ as a random number uniformly distributed in $[-2,2]$ while the total concentrations of adenine nucleotide carriers are normalized. Parameters other than ones obtained from [36] are $[\mathrm{glc}]=20(\mathrm{mM})$, $A_{t}=1(\mathrm{mM}), d=5 \times 10^{-3}\left(\right.$ hour $\left.^{-1}\right), v_{g}=3.6 \times 10^{4}\left(\mathrm{mM}^{-2} \cdot \mathrm{sec}^{-1}\right)$ and $r=5.0\left(\mathrm{mM}^{-1}\right)$.

is a very slow process and represented by a single parameter. The dilution and degradation terms are omitted in the equations of AMP, ADP, and ATP because the de-novo synthesis of the adenine nucleotide carriers is not modeled in the $E$. coli core model. This assumption is equivalent to suppose the homeostasis of the total adenine nucleotide carriers. (We check that the assumption can be relaxed by introducing a phenomenological reaction for the de-novo synthesis of AMP, see SI Text Section.8). According to the growth reaction which we have introduced above, our model cell grows as the reaction (Erythrose 4-phosphate) + (L-Glutamine) $+($ ATP $) \rightarrow($ ADP $)$ proceeds. We chose the simplest kinetics of the growth reaction given by $J_{g}=v_{g}[\mathrm{e} 4 \mathrm{p}][\mathrm{g} \ln ][\mathrm{atp}]$ and the growth rate as $\mu=r J_{g}$. We fit the values $v_{g}$ and $r$ so that the growth rate at the steady-state is in the range of the typical growth rate of $E$. coli in minimal glucose media, in the order of 0.1 per hour, and set the spontaneous degradation rate $d$ as approximately one-hundredth of it. The concentration of the nutrient $([\mathrm{glc}])$ and the total concentration of the adenine nucleotide carriers $\left(A_{t}\right)$ are set to $20 \mathrm{mM}$ and $1 \mathrm{mM}$, respectively (see SI Text Section.1).

\section{Dormant trajectory}

We first computed the steady-state concentrations by simulating the ODE model from random initial conditions. As far as we have checked, there is only a single steady-state. Then, for emulating the exposure to the sub-lethal stresses which disturb intracellular states, we applied random perturbations to the steady-state concentration. The initial concentration of each chemical is given by $10^{u_{i, n}}\left[X_{i}^{(\mathrm{ss})}\right]$. Here $\left[X_{i}^{(\mathrm{ss})}\right]$ and $u_{i, n}$ represents the steady-state concentration of the $i$ th metabolite and a random number for the $i$ th metabolite and $n$th perturbation sampled from uniform distribution in $[-2,2]$. The concentrations of ATP, ADP, and AMP are normalized so that the total concentration is identical for all the initial conditions.

We found that the model exhibited two qualitatively distinct relaxation behaviours depending on the 


\footnotetext{
${ }^{2}$ The concentrations of several chemicals are smaller than 1 molecule per cell, especially in the bottom panel. We discuss the interpretation later
}

initial conditions. The typical time course of each type is plotted in Figs. $2 \mathrm{~A}$ in $\log$ scale, to depict the wide range of the concentration and timescale. Even though the two trajectories eventually relax to the same steady state, the relaxation behaviours are evidently distinct. First, the concentrations after minutes ( 10 ${ }^{-1}$ hours) are different between the top and bottom panel in many orders of magnitude ${ }^{2}$. Also, the characteristic timescale between them is clearly different. The concentrations of the chemicals reach close to the steady values in minutes in the top panel, while the concentrations keep changing for a much longer time, $t \approx 10^{3}$ hours in the bottom panel, which is experimentally indistinguishable from cells stop growing. When sampled over various initial conditions, the distribution of the relaxation time has a clear bimodality as shown in Fig. 2B. Here, the relaxation time is defined as the time when the distance between the steady-state attractor and the state in the logarithm-converted phase space first becomes less than 0.05.

For visualizing the differences among the trajectories, we analyzed all the trajectories in the phase space by the principal component analysis (PCA, see Materials and Methods) where all the trajectories are converted to the logarithmic scale. We plotted all trajectories projected onto the 2-dimensional principal component space (PCS) in Fig. 2C. The trajectories were classified into two groups by the relaxation time and differently colored. The first group is quickly-relaxing trajectories that the trajectory in the top panel of Fig. $2 \mathrm{~A}$ belongs to (colored in green-white-purple). The trajectory in the bottom panel of Fig. 2A is grouped into the other group, colored by blue-white-red which takes a much longer time to relax to the steady-state attractor.

The remarkable gaps between the timescale of chemical reactions, and accordingly, the growth rate during their relaxations highlight the difference between two time courses. The specific growth rate $\mu$ at the steadystate is $\approx 0.5$ hour $^{-1}$ and the model cell achieves this growth rate in a few seconds in the top panel of Figs. $2 \mathrm{~A}$, while less than $10^{-10}$ hour $^{-1}$ in the bottom panel at $t=10^{2}$ hours (at plateau). Thus, in the following sections, we call the trajectories of the second group "dormant trajectories" because of their extremely slower growth rate than that of the other group. Accordingly, the trajectories of the first group are termed "growth trajectories". The following sections are devoted to unveiling the mechanism leading to the differentiation of the growth and dormant trajectories.

\section{Simplification of reaction kinetics}

The kinetic E. coli core model is, as it is, too complicated to understand the mechanism that leads to the two distinct relaxation trajectories. Thus, we simplified the E. coli core model as follows. First, we modified the kinetics of the chemical reactions from the Michaelis-Menten formula to the mass-action rate equation. The rate of the $i$ th chemical reaction $A \rightleftharpoons B, J_{i}$ which was given by

$$
J_{i}=v_{i} \frac{[A]-k_{i}[B]}{1+[A] / K_{A}^{(i)}+[B] / K_{B}^{(i)}}
$$

is replaced by

$$
J_{i}=v_{i}\left([A]-k_{i}[B]\right),
$$

where $v_{i}$ and $v_{i} k_{i}$ are the rate constant of forward- and backward reaction, respectively. Note that the massaction kinetics (Eq.(3)) is a special form of the Michaelis-Menten kinetics (Eq.(2)) in the parameter region where $[A] \ll K_{A}^{(i)}$ and $[B] \ll K_{B}^{(i)}$ hold (for general arguments, see [42]). The model is then nondimensionalized by scaling the concentrations by the external glucose concentration and the time by the rate constant of the glucose uptake. We further simplified the rate equations by setting $v_{i}$ 's to unity and binarising $k_{i}$ 's for all $i^{\prime}$ s. The $E$. coli core model contains the information of irreversibility for each reaction, and thus, if the $i$ th reaction is reversible, we set $k_{i}$ as unity, and otherwise, set it to $\kappa \ll 1$. We term this simplified version of the kinetic $E$. coli core model as model0 with an index for the following model reduction steps.

Surprisingly, the emergence of distinct relaxation trajectories is robust to such an extensive modification of the model. The qualitative difference of the trajectories (Figs. S1A), bimodality of the distribution of the relaxation time (Fig. S1B), and the distinction of the trajectories in the PC1-PC2 space (Fig. S1C) were 
unchanged. This robustness implies that the emergence of the distinct trajectories stems from the structure of the metabolic reaction network of the $E$. coli core model, rather than choices of specific parameter values. We also confirmed that the distinct trajectories emerge if the kinetic parameters are randomly assigned instead of setting them unity (see SI Text Section.11).

Now it is worth asking, if there are understandably simple, minimal network architecture(s) in the $E$. coli core network which leads to the distinct trajectories. For extracting the architectures, we develop a method of systematic network reduction in the following section.

\section{Systematic Model Reduction}

In the present section, we reduce the $E$. coli core network to obtain a minimal network exhibiting the distinct relaxation dynamics. As far as we know, there is no method to reduce the reaction network without losing the characteristic nature of the relaxation dynamics. Once the concentrations of the cofactors are dealt as variables, metabolic reactions in the model get highly interconnected and the well-known reduction method works poorly. For instance, adiabatic elimination may eliminate merely one or few reactions and it is hopeless to obtain an understandably-simple model. Thus, here we focus only on the emergence of the distinct trajectories. As will be seen, this allows us to derive a much simpler model than the original model.

In the following, we remove one or a few reactions from the network step by step and check if the reduced model still exhibits the distinct trajectories (a solid criterion is introduced later). As illustrated in Fig. 3A, we consider two types of reaction removal, namely, the simple removal and the contraction. First, we describe the simple removal. Suppose that there are reactions $A \rightleftharpoons B, B \rightleftharpoons C$ and $C \rightleftharpoons A$, and also $A$ and $B$ are connected to the rest part of the network by the other reactions (Fig. 3A-(i)). The simple removal removes the reaction $B \rightleftharpoons C$ and $C \rightleftharpoons A$, and accordingly, eliminate the chemical $C$ because it is a disconnected component in the network.

In the contrast, chemical species are merged by the contraction (Fig. 3A-(ii)). It removes a reaction $A \rightleftharpoons E$, and then, the chemical $A$ and $E$ are identified forming a new chemical $Æ$. Here, we avoided the appearance of the dead-end chemical which has only one reaction because networks with dead-end chemicals can cause the heavy accumulation of the chemicals and it potentially leads to an artifactual anomalous relaxation behaviour.

At each reduction step, we checked if the reduced model exhibits the two distinct classes of trajectories by computing its dynamics: First, we run the ODE model from 512 randomly generated initial points to search the attractors. Except for a single model, all the intermediate models of the reduction process showed monostability (we will revisit the exception later). Then, 512 initial conditions were generated by perturbing the steady-state concentration in the same way as we did in the previous models.

The bimodality of the relaxation time distribution is one of the best intuitive criteria of the distinct trajectories. However, we found in some cases the bimodality was unclear even though there were clearly different types of trajectories when we plotted the time courses and performed PCA. This is because the growth rates during the relaxation of both types of trajectories (growth and dormant) become smaller than the spontaneous degradation rate $d$, and thus, the relaxation time of all the trajectories becomes approximately $1 / d^{3}$. Thus, we decided to focus on the similarity of the trajectories instead of the relaxation time itself. Below, we first intuitively explain how we quantify the similarity of the trajectories, and then, introduce the actual measure.

Suppose that a model has a single attractor. Then, all the trajectories starting from different initial points eventually converge. We like to categorize the trajectories into different groups in a way that, if a pair of trajectories monotonically approach each other as they converge to the attractor, they belong to the same group. One may naïvely expect that we can state that two trajectories $x(t)$ and $y(t)$ monotonically approach each other if the Euclidean distance between them at the same time point, $d(x(t), y(t))$, is a monotonically decreasing function of $t$. However, since the initial points are distributed in the phase space, measuring the distance between the points on two trajectories at the same time point is unreasonable.

\footnotetext{
${ }^{3}$ In principle, we can overcome this problem by setting $d$ to be sufficiently small such as $10^{-20}$. However, it requires ridiculous amounts of computation. The distributions of the relaxation time for all the models in the reduction step are presented in $S I$ Text Section.3
} 
A

(i). simple removal

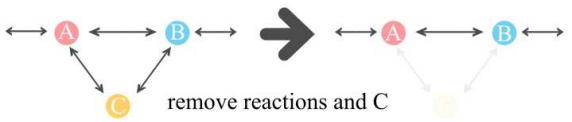

(ii). contraction

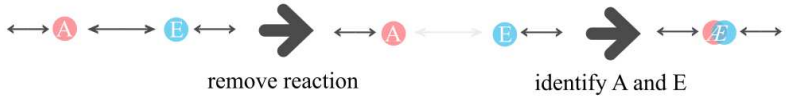

C
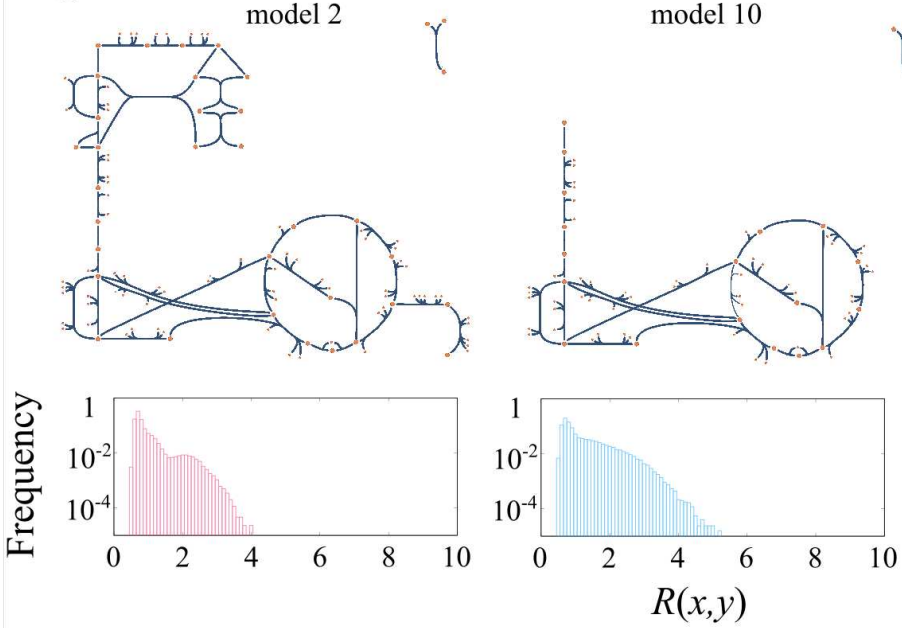

model 10
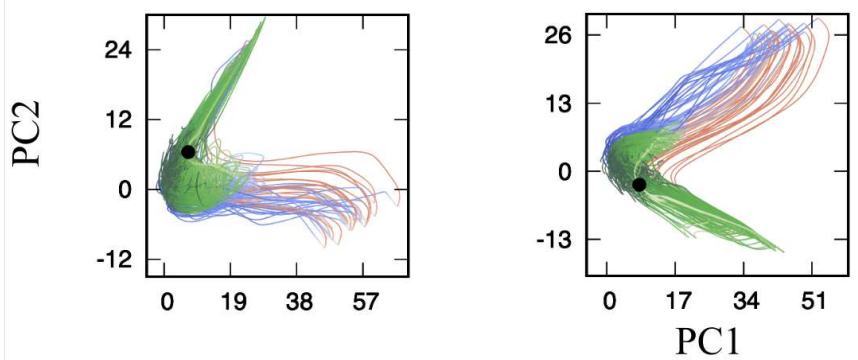

B
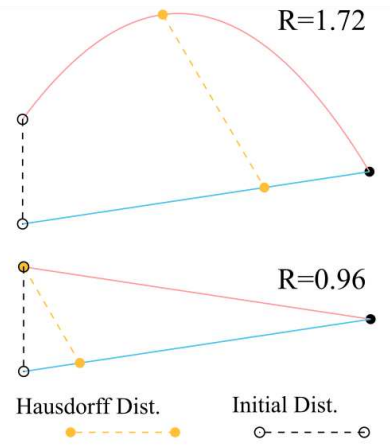

model 16
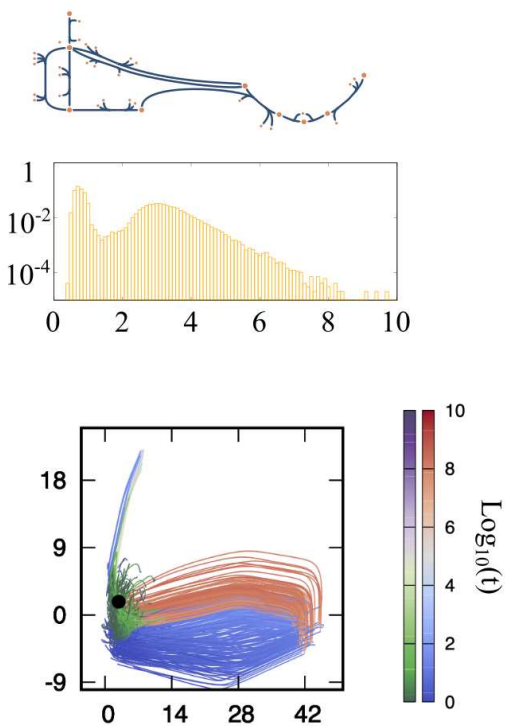

Figure 3: A. Two types of reaction removal. The simple removal (top) just removes one or a few reactions from the network. The number of reactions to be removed is determined so that the dead-end chemicals are not made by the removal. The contraction removes a single reaction first, and then, the substrate and product of the removed reaction are identified and regarded as a new chemical species. B. Illustrative description of the Hausdorff distance and the expansion ratio. There are two cases are depicted: the case with $R>1$ (top) and $R<1$ (bottom). The two lines (pink and cyan) are trajectories starting from the initial point of each (back circles), converging to the same attractor (black dot). The length of the yellow dashed line and the black dashed line give the Hausdorff distance and the initial distance, respectively. If one trajectory (pink) goes away from the other (blue), typically the Hausdorff distance becomes larger than the initial distance. In the contrast, in the case that the two trajectories approach each other, the Hausdorff distance is smaller than the initial distance. Note that the information of time is discarded in the argument. C. The reduced networks of the intermediate models (models 2, 10, and 16) are drawn. The sole nutrient (glucose) is at the left top corner of the network. The distribution of the expansion ratio and the trajectories projected onto the PCS of each model are also plotted. For the coloring protocol of the trajectories, see the main text. 
Thus, instead of adopting this naïve definition of the monotonicity, we measure the maximum Euclidean distance between two trajectories in the phase space. It is known as the Hausdorff distance of the trajectories, given by

$$
d_{H}(x, y)=\max \left\{\max _{t} \min _{s} d(x(t), y(s)), \max _{t} \min _{s} d(x(s), y(t))\right\} .
$$

The Hausdorff distance first looks for the closest point of the trajectory $y$ from the point $x(t), y\left(s^{*}(t)\right)$, and then find the pair of the points $\left(x(t), y\left(s^{*}(t)\right)\right)$ which gives the maximum Euclidean distance. The same is done from the points of $y(t)$ and the larger value is chosen for the symmetry $d_{H}(x, y)=d_{H}(y, x)$. The Hausdorff distance thus measures how far the two trajectories are distant while trivially distant pairs of points are not taken into account (for example, the initial point of $x$ and the endpoint of $y$, i.e, the attractor).

We cannot judge whether the trajectories go away from each other or not directly from the Hausdorff distance since it needs to be compared with the initial separation. Thus, we normalize the Hausdorff distance by the Euclidean distance between the initial points, $d(x(0), y(0))$ leading to

$$
R(x, y)=\frac{d_{H}(x, y)}{d(x(0), y(0))} .
$$

We call this ratio $R(x, y)$ as the expansion ratio of the trajectories $x$ and $y$. It measures how much the initial distance has expanded. If $d_{H}(x, y)$ is smaller than the initial distance, $R(x, y)$ is less than unity ${ }^{4} . R(x, y)>1$ means that two trajectories go away from each other at least once despite eventually converging to the same attractor. The concept of the Hausdorff distance and the expansion ratio are illustrated in Fig. 3B. Note that in this manuscript, the Euclidean distance, and accordingly, the Hausdorff distance are measured in the original high-dimensional phase space after applying the logarithm-conversion of the variables, not in the lower-dimensional principal component space.

The distribution is expected to have a trivial peak around $R=1$. If the distribution has only a trivial peak, it indicates that all the trajectories are monotonically attracted to a single predominant stream in the phase space reaching the attractor. Oppositely, if the distribution has a non-trivial peak(s) and/or an additional long-tail, then it means that the correlation between the initial distance and the Hausdorff distance is not simply scaled to each other. Therefore, in the present manuscript, we utilize the multimodality and/or the long-tail of the distribution of the expansion ratio as the criterion of the distinct trajectories (examples can be found in Fig. 3C). We examine if the model has exhibited distinct trajectories by fitting the distribution by a sum of Gaussian functions. In rough terms, it checks if the distribution needs at least two Gaussian functions with distant peaks (see SI Text section.2 for the details). Note that in the following analysis, the computation of the expansion ratio and PCA were performed for the trajectories converted to the logarithmic scale so that the dynamic behaviours of the chemicals with low concentrations are also reflected in the analysis ${ }^{5}$.

\section{A Minimal Model}

We have reduced the E. coli core model step-by-step according to the model reduction method described in the previous section. For accomplishing the network reduction, we manually determined the order of the reaction removal so that subsystems of the network are removed or contracted in consecutive reduction steps. We completed the model reduction by removing and contracting the L-glutamine synthesis pathway (4 steps), pentose-phosphate pathway (4 steps), glycolytic pathway (3 steps), and TCA cycle (7 steps) with the indicated number of steps in the parenthesis. The full list of the removed reactions is provided in SI Data.1. Also, the distributions of the expansion ratio and the trajectories on PCS of all the models are shown in SI Text Section.3. Note that we also tried the model reduction in random orders of the reaction removal (see SI Text Section.10). The minimal networks led by the reduction surely depend on the order of the reaction removal. However, all the minimal networks commonly satisfied the two conditions for the emergence of the distinct trajectories discussed later. We revisit the case of random-order reduction in the Discussion section.

\footnotetext{
${ }^{4} R$ becomes smaller than 1 if there is any point on $x$ which is closer to $y(0)$ than $x(0)$. This argument is symmetric for the swap of the indices.

${ }^{5}$ As a side effect of the logarithm-conversion, the behaviours of the chemicals with quite low concentrations may be too much highlighted. We computed the expansion ratio with cut-offs of the concentrations for a lower-side, see $S I$ Text section.4
} 
The reaction network, the distribution of the expansion ratio, and the trajectories projected onto the PCS of selected models are shown in Figs. 3C. The distributions of the expansion ratio show clear bimodality or the long tail. We colored the trajectories based on the relaxation time of each. The figure shows that dormant trajectories (blue-white-red trajectories) commonly take detours to reach the attractor in the PCS. This is not an artifact due to the PCA. We computed the ratio of the line integral of the trajectory $(L)$ to the Euclidean distance between the initial point and the attractor $(D)$ in the original high-dimensional phase space for each trajectory. The average $L / D$ ratio of the dormant trajectories was indeed bigger than that of the growth trajectories (SI Text Section.5). We found only the 8th model showed bistability (i.e, two attractors). In the analysis, we chose the major attractor that approximately $92 \%$ of the randomly-generated initial points converging. We checked all the initial points generated by the perturbation of this attractor returned back to the attractor. Note that the trajectory starting from perturbed points from the other, minor attractor, also showed a bimodal distribution of the expansion ratio (see SI Text Section.6).

After the 18 steps of reductions, we reached the stage that no more reduction is possible without losing the multimodality of the distribution of the expansion ratio. The reaction network and reaction names that remained in this minimal network (model 18) are depicted together with the original E. coli core network in Fig. 4A. The network consists of glucose (glc), phosphoenolpyruvate (pep), pyruvate (pyr), oxaloacetate (oaa), ATP, ADP, and AMP. As highlighted in the original network, the reaction from glc to pep is the contraction of the glycolytic pathway and oaa is representative of the chemicals in the TCA cycle. It is worth noting that the local structure of the network among pep, pyr, and oaa are unchanged (cyan boxes). In other words, the minimal network is obtained by removing the pentose phosphate pathway and contracting the glycolytic pathway and the TCA cycle. Also, the reaction ADK1 converting two ADPs to ATP and AMP is conserved. As shown in Fig. 4B and C, the model still exhibits distinct trajectories.

The model consists of five variables (recall that [glc] and [atp] + [adp] + [amp] are constant). In order to simplify the model further, we adiabatically solved the concentrations of three chemicals, [atp], [adp], and [oaa], under given values of [pep] and [pyr] to eliminate these variables from the model. This adiabatic elimination of the three variables does not violate the multimodality of the distribution of the expansion ratio. The set of ODEs of the minimal model is then given by

$$
\begin{aligned}
\frac{d[\text { pep }]}{d t} & =-([\text { atp }][\text { pep }]-[\text { adp }][\text { glc }]) \\
& -([\text { pep }]-\kappa[\text { oaa }])+([\text { atp }][\text { pyr }]-\kappa[\text { amp }][\text { pep }]) \\
& -([\text { adp }][\text { pep }]-\kappa[\text { atp }][\text { pyr }]) \\
& -(d+r[\text { oaa }][\text { atp }])[\text { pep }], \\
\frac{d[\text { pyr }]}{d t} & =-([\text { atp }][\text { pyr }]-\kappa[\text { amp }][\text { pep }]) \\
& +([\text { adp }][\text { pep }]-\kappa[\text { atp }][\text { pyr }]) \\
& -(d+r[\text { oaa }][\text { atp }])[\text { pyr }],
\end{aligned}
$$

where [atp], [adp] (accordingly [amp]), and [oaa] are adiabatically solved, and thus, are the functions of [pep] and $[\mathrm{pyr}]$.

The two-variable system allows us to visualize the vector field. As shown in Fig. 4D, interestingly, there is a boundary below and above which the streamlines change the direction dramatically ([pep] $\lesssim 0.1$ and $[\mathrm{pyr}] \approx 1)$. Below the boundary, the state relaxes to the attractor rather straightforwardly corresponding to the dynamics shown in Fig. 4B (growth trajectory). On the other hand, trajectories starting from the upper region first travel to the left side of the phase space (small [pep] region) and return back to the attractor, corresponding to Fig. 4C (dormant trajectory). We attribute the emergence of the distinct trajectories to this dramatic change of the directions of the vector field occurring across the boundary. Hereafter, we call the region above- and below the boundary as the dormant and the growth region, respectively. 
A

\section{Reaction List}

$\begin{array}{ll}\text { Uptake } & \text { glc }+ \text { adp } \rightleftarrows \text { pep }+ \text { atp } \\ \text { PYK } & \text { pep }+ \text { adp } \rightarrow \text { pyr }+ \text { atp } \\ \text { PPS } & \text { pyr }+ \text { atp } \rightarrow \text { pep }+ \text { amp } \\ \text { PPC } & \text { pep } \rightarrow \text { oaa } \\ \text { Growth } & \text { oaa }+ \text { atp } \rightarrow \text { adp } \\ \text { ADK1 } & \text { atp }+ \text { amp } \rightleftarrows 2 \text { adp }\end{array}$
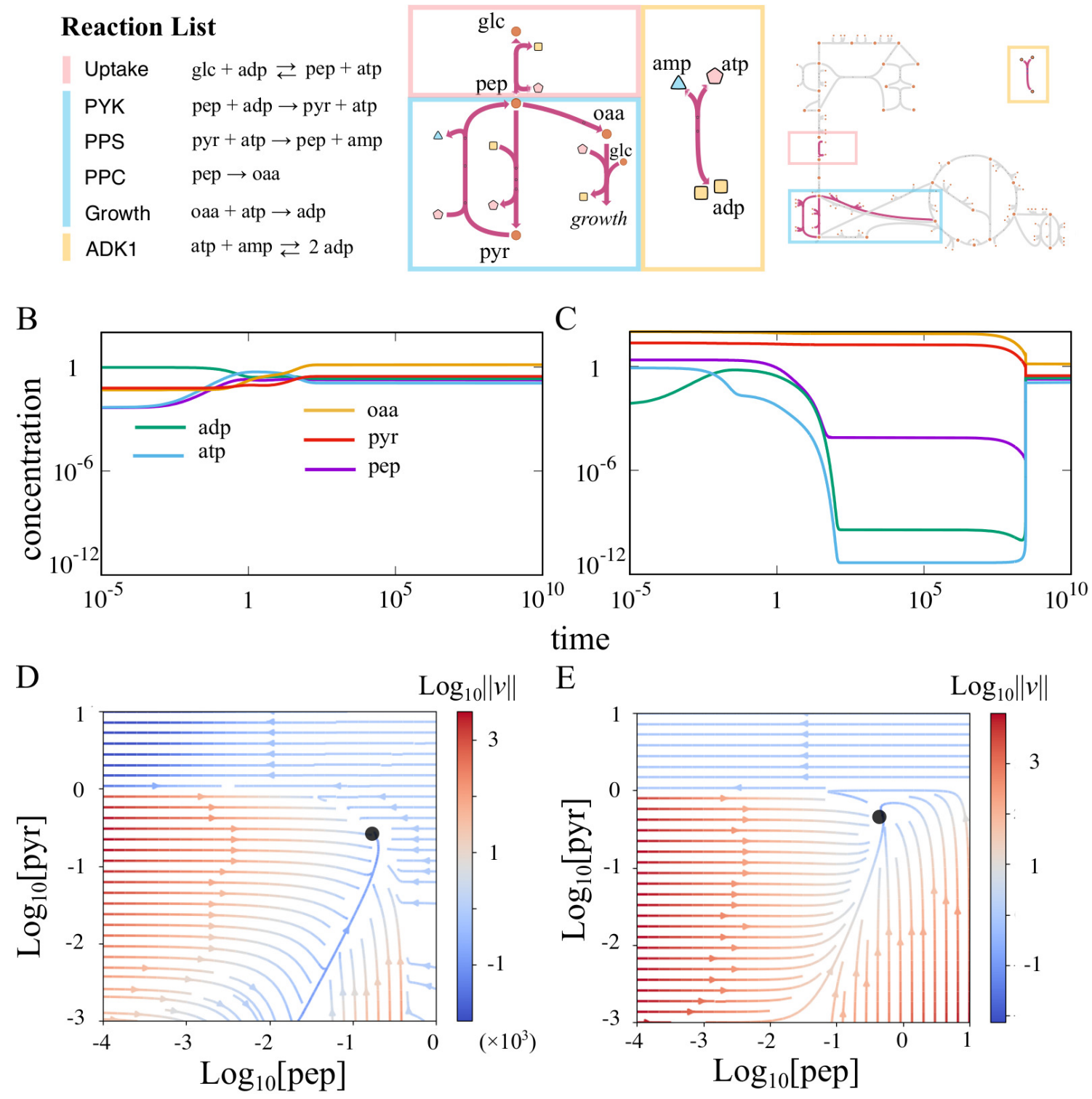

Figure 4: A. The list of the reactions in the minimal network (left). The structure of the minimal network (middle). The original network with the reactions in the minimal model is highlighted (right). The minimal model consists of three parts, namely, the glycolytic pathway (pink bar and boxes), the joint part between the glycolytic pathway and the TCA cycle (cyan bar and boxes), and the adenosine kinase reaction (yellow bar and boxes). B and C. Example time courses of the growth trajectory (B) and the dormant trajectory (C) of the minimal model. D. The streamline representation of the vector field of the 2-variable minimal model where the steady values of [atp], [adp] and [oaa] under given [pep] and [pyr] are numerically solved. E. The streamline representation of the simplified minimal model. The color indicates the norm of the vector $v=(d[\mathrm{pep}] / d t, d[\mathrm{pyr}] / d t)$ at each point and the black dots indicate the attractor of each in $\mathrm{D}$ and $\mathrm{E}$. $\phi_{0}=10^{-8}$ in $\mathrm{E}$. 


\section{Conditions for the emergence of distinct trajectories}

What determines the boundary between the growth- and the dormant region, and why is the vector field of the 2-variable model (Fig. 4D) almost parallel to the horizontal axis in the dormant region? For the first point, we found that there are large gaps in the concentrations of ATP and ADP between the two regions. In the dormant region, their concentrations are low $\left(\sim 10^{-8}\right)$ while in the growth region, they are in the order of 0.1. This gives an insight into the second point. We found that the drastic change of the direction of the vector field occurs because four of the five reactions of the model coupled with the adenine nucleotide carriers, and thus, these reactions almost completely halt in the dormant region.

Intuitively, the low level of ATP and ADP in the dormant region can be understood from the reactions in Fig. 4A as follows. First, let us consider the situation where the concentrations of pep and pyr are in the dormant region. If in addition, the concentration of pep is low, the uptake reaction proceeds in the direction glc + adp $\rightarrow$ pep+atp. When this reaction accumulates some ATP, PPS proceeds in the direction pyr+atp $\rightarrow$ pep+amp, because the concentration of pep is low. In total, the uptake reaction and PPS form a futile cycle that converts ATP and ADP into AMP. Note that the PPS does not easily result in the accumulation of pep, because of PPC reaction. If the concentration of pep is high but pyr is also high so that the system is still in the dormant region, PYK plays the same role as the uptake reaction in the previous case. Thus, a futile cycle converting ATP and ADP into AMP is formed in both cases. If the conversion from ATP and ADP into AMP is slow enough, the other reaction, ADK1 (atp+amp $\rightleftharpoons 2 \mathrm{ADP}$ ) proceeds to balance $[\mathrm{atp}]+[\mathrm{adp}]$ and $[\mathrm{amp}]$. However, ADK1 cannot balance them if the conversion is too fast because the reaction needs ATP.

Indeed, this intuitive description is consistent with the analytical estimate of the boundary. For that, we derive the boundary between the growth and dormant region for [pep] $\ll 1$. The boundary is given by the concentration of pyr leading to a low concentration of ATP and ADP with a given value of [pep]. For the estimation of the critical [pyr], we sum up $d[\operatorname{atp}] / d t$ and $d[\operatorname{adp}] / d t$ and assume [atp], [adp], [pep] $=O(\epsilon)$ with $\epsilon \ll 1$. Also, recall that the irreversibility parameter $\kappa$ in (Eq. (6)) is small. Then we obtain

$$
\frac{d([\operatorname{atp}]+[\operatorname{adp}])}{d t}=J_{\mathrm{ADK} 1}-J_{\mathrm{PPS}} \sim[\operatorname{atp}]\left(v_{\mathrm{ADK} 1} A_{t}-v_{\mathrm{PPS}}[\mathrm{pyr}]\right),
$$

where we explicitly write down the rate parameter $v_{*}$ 's and the total concentration of adenine nucleotide carriers $A_{t}$ for the interpretation of the estimate. If the first term of the right most side is larger than the second term, $[\mathrm{atp}]+[\mathrm{adp}]$ increases, while in the opposite situation the sum keeps decreasing to zero as long as [atp] is non-zero. This shift occurs at $v_{\mathrm{PPS}}[\mathrm{pyr}] \sim v_{\mathrm{ADK} 1} A_{t}$, and it gives the boundary between the growing and the dormant regions.

Next, we explain how the decrease of [atp] and [adp] leads to the vector field parallel to the horizontal axis in the dormant region (Fig. 4D). Let us assume that the concentrations of ATP and ADP are approximately the same and well-represented by a single lumped parameter $\phi$. Also, for a simplicity, we set the irreversibility parameter $\kappa$ to zero. Then, the ODE for the 2-variable minimal model (Eq. (6)) is given by

$$
\begin{aligned}
& \frac{d[\mathrm{pep}]}{d t}=\phi(1-[\mathrm{pep}]+[\mathrm{pyr}]+r[\mathrm{oaa}])-(1+d)[\mathrm{pep}] \\
& \frac{d[\mathrm{pyr}]}{d t}=\phi([\mathrm{pep}]-[\mathrm{pyr}]+r[\text { oaa }])-d[\mathrm{pyr}]
\end{aligned}
$$

where [oaa] is the function of $\phi$, while it becomes constant as $\phi$ approaches 0 . From the equation, we can see that if the concentration of ATP and ADP, represented by $\phi$, is $O(1)$ (i.e, in the growth region), the timescale of the system is $O(1)$. On the other hand, if ATP and ADP deplete and $\phi \approx 0$ holds in the dormant region, the timescale of $d[\mathrm{pyr}] / d t$ becomes $O(d)$. Since the spontaneous degradation rate $d$ is sufficiently smaller than unity, $|d[\mathrm{pep}] / d t| \gg|d[\mathrm{pyr}] / d t|$ holds, and it leads to the vector field being almost parallel to the [pep] axis as depicted in Fig. 4D.

To confirm if the simplification above still captures the feature of the vector filed in Fig. $4 \mathrm{D}$, we have drawn the vector field of the simplified model Eq.(9) and (10) with $\phi=\max \left\{1-[\mathrm{pyr}], \phi_{0}\right\}$ in Fig. 4E. It well captures the feature of the original vector field. We have confirmed that the shape of the vector field is 
robust to the choice of the function $\phi$. Also, we analytically solved the model without the growth dilution term (Eq.(9) and (10) with $r=0)$ and found that the model has only a single timescale which is $O(1)$ in the growth region (see SI Text Section.7).

The simplified model (Eq.(9) and (10)) highlights that the timescale of $d[\mathrm{pep}] / d t$ is much faster than that of $d[\mathrm{pyr}] / d t$ in the dormant region. The right hand side of Eq.(9) has the term $(1+d)[\mathrm{pep}]$, while that in Eq.(10) is only the degradation term $d[\mathrm{pyr}]$, and this difference results in the parallel streamline in the phase space (Fig. $4 \mathrm{E})$. It is worth noting where the term $(1+d)[\mathrm{pep}]$ in Eq. $(9)$ comes from. $d$ corresponds to the constant-rate degradation term, and the reactions coupled with either ATP or ADP should have the rate being proportional to $\phi$. Therefore, this timescale 1 comes from the reaction coupled neither with ATP nor ADP, namely, PPC (pep $\rightarrow$ oaa). All the reactions except PPC are coupled with either ATP or ADP, and thus, the reactions slow down over the boundary between the growth- and the dormant region. However, the rate of PPC has no direct effect from the depletion of ATP and ADP. Then, even after the slowing down of almost all reactions, pep is kept being consumed, and it leads to the characteristic dynamics of the dormant trajectory.

Note that, if PPC were also coupled with ATP and ADP, $(1+d)$ [pep] term in Eq.(9) would have been replaced by $(\phi+d)[\mathrm{pep}]$. In such a case, all the reactions would have been uniformly slowed down by the depletion of ATP and ADP, and the direction of the vector field would not change over the boundary as drastically as Fig. 4D. Thus, it is vital that the reaction system partially slows down due to the depletion of ATP and ADP.

It is noteworthy that the network structure is also a part of the mechanism: if PPC were the reaction converting pyr to oaa instead of pep to oaa, the drastic change of the direction of the vector field as Fig.4D would not result. If PPC were pyr $\rightarrow$ oaa, the main part of the reaction network (reactions except for ADK1) would have no branch. The slowing down of the upstream reactions of PPC (i.e, uptake, PYK, and PPS) would be rate-limiting steps of it and PPC would slow down coordinated with these reactions.

The above two points may suggest that large discrepancies of the chemical concentrations between the steady-state and the plateaux lead to distinct dynamics. In both cases-PPC with energy coupling and the main network without a branch-the reactions uniformly slow down. In such scenarios, even if ATP and ADP deplete, the difference between production and consumption of each chemical stays relatively small, and thus, the changes of the concentrations remain small. However, if the slowing-down occurs heterogeneously on the network, some chemicals will have a large mismatch between production and consumption. As a consequence, the concentrations of such chemicals drastically change from the concentrations before the depletion of ATP and ADP.

To sum up, the mechanism of the emergence of the distinct trajectories has two parts: (i) the unbalance of energy (ATP and ADP) production and consumption, and (ii) partial slowing-down of the reaction system caused by non-uniform coupling to the energy currencies and branching of the network.

\section{Discussion}

We have shown that E.coli central carbon metabolism exhibits distinctly different dynamics depending on the perturbation from the steady-state concentration. The two types of trajectories greatly differed in terms of the relaxation time and the growth rate during the relaxation, and thus, we termed them as the growthand the dormant trajectories. We developed a systematic method to simplify the reaction network without losing the distinct trajectories. By the successive reduction of the model, we eventually reached the minimal network still exhibiting the qualitatively same behavior.

By drawing the vector field of the 2-variable minimal model, we found that there is a boundary at which the vector field changes the direction drastically. Indeed, the two regions are divided by the boundary corresponding to the set of the initial points of the growth- and the dormant trajectories. The analysis led that there are at least two vital requirements for the distinct trajectories: (i) the unbalance of the energy production and consumption and (ii) the partial slowing-down of the reactions due to the non-uniform coupling with the energy currency molecules and branching of the network.

We carefully examined the robustness of our main results, namely, the emergence of distinct trajectories and the consequence of model reduction by studying several model variants. First, the robustness of the emer- 
gence of distinct trajectories to the parameter values was examined. For several values of the total adenine nucleotide carriers concentration $\left(A_{t}\right)$, we randomly assigned the rate constant $\left(v_{i}\right)$ and the irreversibility constant $\left(k_{i}\right)$ of each reaction (Eq.(3)) and studied if the distribution of the expansion ratio is multimodal and/or long-tailed. As anticipated from the analysis, if the total concentration, $A_{t}$, is too large for the depletion of ATP and ADP to occur, the expansion ratio becomes unimodal (SI Text Section.11). Otherwise, the multimodality was robust to the parameter choice. We confirmed that the model without the replacement of the nicotinamide nucleotide carriers by the adenine nucleotide carriers exhibits distinct trajectories (SI text Section.9). Also, we have confirmed that the distinct trajectories emerge if the assumption on the constant total concentration of the adenine nucleotide carriers is relaxed by introducing a phenomenological reaction for the de-novo synthesis of AMP to the minimal model (SI Text Section.8). Overall, the emergence of distinct trajectories is a robust feature of the E.coli core model rather than a phenomenon led by fine-tuning of the parameters.

To confirm if the requirements are not limited to the specific model reduction example, we performed the reaction removal for the model reduction in 16 different randomized orders. All the minimal networks obtained by the random reduction were larger than those derived in the result section. Interestingly, we found that there are differences in the network structure depending on whether the distribution of the expansion ratio shows the clear multimodality or only the long tail. The minimal models with the clear multimodal distribution share two features: (a) the models keep ADK1 reaction and $\mathrm{AMP}^{6}$, and (b) in each network, there are both reactions, with- and without the coupling to the energy currency molecules (ATP, ADP, and AMP) as well as branches. The shared features correspond to the requirements above. However, in the minimal models with only the long-tail, all the reactions are coupled with the energy-currency molecules, i.e, the above feature (b) is absent. We also found that the total concentration of ATP and ADP plays a central role in the emergence of distinct trajectories (see SI Text Section.5). Overall, our results shed light on the impact of the cofactors' role on the dormant transition from the mathematical modeling point-of-view, supporting the hypothesis presented in $[16,18]$.

The present model showed a possibility that the dormancy transition could be triggered by the metabolic dynamics itself when the metabolic state is perturbed. Here we succeeded to highlight the role metabolic dynamics because in the present model we set the enzyme level constant based on the clear time-scale separation between the metabolic reactions and modulations of the protein level [21,22]. According to what the minimal model tells us, the perturbation evokes the futile cycle and leads to the depletion of ATP and ADP. Here, the sources of the perturbation can be starvation, nutrient shift, exposure to antibiotics, $\mathrm{pH}$ stress, or even stochasticity of the intracellular processes.

However, the intracellular states of the dormant cells, in reality, may go beyond what we can depict in terms of metabolites. For instance, the ATP depletion can lead to the protein aggregation [19,20]. Also, disturbances of metabolic states may lead to the stress-response systems being controlled by (p)ppGpp, toxin-antitoxin modules, and the alternative sigma factor $\sigma^{S}$ [10-15]. Therefore, the present result suggest a mathematical mechanism of the transition to the dormancy, while for developing a mathematical theory of the cells after the transition, we need to extend the model so that the dynamics of gene expression and protein concentrations are included. This is one of the reasons why the concentrations of some chemicals went below a single molecule per cell $(\approx 1 \mathrm{nM})$ in the dormant dynamics (Fig.2A). The ATP level actually depletes also in the real cell during the dormant transition, while too strong depletion like what the present model exhibited may be prevented by regulations ${ }^{7}$.

Note that we can find the counterpart of the reactions in the minimal model in the full E.coli metabolic network, and thus, the dormancy transition demonstrated by the minimal model is verifiable by experiments. The central part of the mechanism is that PPS and PYK can form the futile cycle and the competition between PPS and ADK1 on the consumption/production of ATP. Indeed, the experiments showed that one

\footnotetext{
${ }^{6}$ Note that the model reduction algorithm tried to remove the reaction, while the removal was rejected because the distribution of the expansion ratio of the derived models exhibited neither multimodality nor long-tail.

${ }^{7}$ Also in growth dynamics, the concentration of a chemical species which is one of the growth factors, glutamine, becomes lower than 1nM. We attribute this to the technical reason: the gap in the definition of the growth reaction (the biomass synthesis reaction) between the present model and the model in the paper where the kinetic parameters were estimated [36]. In the model studied in [36], the biomass synthesis reaction was not incorporated into the model. Therefore, glutamine is consumed much faster in the present model than the model for the parameter estimation.
} 
can induce the ATP-consuming futile cycle between phosphoenolpyruvate and pyruvate via PYK and PPS by overexpressing the ppsA gene $[43,44]$. Taken the experimental reports and the present computational results together, we can hypothesize that the overexpression of the pps gene leads to an increase of the persister fraction because PPS converts ATP to AMP.

Lastly, we like to remark that the model reduction method developed in the present manuscript can be applied also to the metabolic models of other organisms. We can find several reactions which potentially form a futile cycle from a variety of species. For instance, each of Acetyl-CoA synthetase (KEGGID:R00235), Phosphoribosylpyrophosphate synthetase (R01049), and Asparagine synthase (R00578) [45] converts ATP to AMP and forms a loop in the metabolic networks. These are the minimum requirements for a reaction to form a futile cycle discussed above. Such reactions are widespread from prokaryotes to eukaryotes, and from unicellular to multicellular organisms. Comprehensive studies of the kinetic models of not only E. coli but also other organisms may pave a way for understanding the robust and generic network features leading to the multiple timescales of cellular growth and dormancy.

\section{Acknowledgments}

The authors thank Chikara Furusawa and Sandeep Krishna for the fruitful discussion. This work is supported by the research grant (00028054) from VILLUM FONDEN.

\section{Materials and Methods}

\section{Simulation of ordinary differential equations (ODEs)}

All the ODE computations were performed by using Matlab (Mathworks) ode23s function. For searching attractors, we set $10^{u_{i, n}}$ for the $i$ th metabolite as the $n$th initial value where $u_{i, n}$ is the random number generated from a uniform distribution in $[-1,1]$. The steady-state concentration $\left[X_{i}^{(\mathrm{ss})}\right]$ is then obtained, initial concentrations for the main analysis of the dynamics are generated as $10^{u_{i, n}}\left[X_{i}^{(\mathrm{ss})}\right]$ with $u_{i, n}$ is the same random number yet distributed in $[-2,2]$. The ODEs were computed with two tolerance options $\left(\right.$ AbsTol $\left.=10^{-10}, \operatorname{RelTol}=10^{-12}\right)$ and $\left(\right.$ AbsTol $=10^{-10}$, RelTol $\left.=10^{-14}\right)$ from exactly the same initial points. After the computation, the trajectories with two different RelTol values, but from the same initial point were compared for the quality check of the computation. If the Hausdorff distance of the pair of the trajectories was less than 0.5 , the trajectories were considered as correctly computed and the trajectory obtained with RelTol $=10^{-14}$ was used for the further analysis, and otherwise, discarded. The quality check of the computation was performed after the transformation $x(t) \rightarrow \ln (x(t))$ where $x(t)$ is the concentration of the chemicals.

\section{Hausdorff distance}

Hausdorff distance is computed by using the python package scipy.spatial.distance.directed_hausdorff as the maximum of two directed Hausdorff distances after the transformation of concentration into the natural logarithm of the concentration.

\section{Principal Component Analysis}

We used the python package sklearn.decomposition.PCA [46] without whitening. The whitening leads to only a minor effect on the results. The concentrations of the chemicals were transformed into the natural logarithm of the concentration before the analysis. 


\section{References}

[1] Guennadi Sezonov, Danièle Joseleau-Petit, and Richard d'Ari. Escherichia coli physiology in luria-bertani broth. Journal of bacteriology, 189(23):8746-8749, 2007.

[2] Nathalie Q Balaban, Jack Merrin, Remy Chait, Lukasz Kowalik, and Stanislas Leibler. Bacterial persistence as a phenotypic switch. Science, 305(5690):1622-1625, 2004.

[3] Nathalie Q Balaban, Sophie Helaine, Kim Lewis, Martin Ackermann, Bree Aldridge, Dan I Andersson, Mark P Brynildsen, Dirk Bumann, Andrew Camilli, James J Collins, et al. Definitions and guidelines for research on antibiotic persistence. Nature Reviews Microbiology, 17(7):441-448, 2019.

[4] Susanne K Christensen, Marie Mikkelsen, Kim Pedersen, and Kenn Gerdes. Rele, a global inhibitor of translation, is activated during nutritional stress. Proceedings of the National Academy of Sciences, 98(25):14328-14333, 2001.

[5] Yusuke Himeoka and Namiko Mitarai. When to wake up? the optimal waking-up strategies for starvation-induced persistence. PLoS computational biology, 17(2):e1008655, 2021.

[6] Mikkel Skjoldan Svenningsen, Sine Lo Svenningsen, Michael Askvad Sørensen, and Namiko Mitarai. Existence of log-phase escherichia coli persisters and lasting memory of a starvation pulse. Life Science Alliance, 5(2), 2022.

[7] Irit Levin-Reisman, Orit Gefen, Ofer Fridman, Irine Ronin, David Shwa, Hila Sheftel, and Nathalie Q Balaban. Automated imaging with scanlag reveals previously undetectable bacterial growth phenotypes. Nature Methods, 7(9):737, 2010.

[8] Yusuke Himeoka and Kunihiko Kaneko. Theory for transitions between exponential and stationary phases: universal laws for lag time. Physical Review X, 7(2):021049, 2017.

[9] Elena Biselli, Severin Josef Schink, and Ulrich Gerland. Slower growth of escherichia coli leads to longer survival in carbon starvation due to a decrease in the maintenance rate. Molecular Systems Biology, 16(6):e9478, 2020.

[10] Jakub Leszek Radzikowski, Silke Vedelaar, David Siegel, Álvaro Dario Ortega, Alexander Schmidt, and Matthias Heinemann. Bacterial persistence is an active $\sigma$ s stress response to metabolic flux limitation. Molecular systems biology, 12(9):882, 2016.

[11] Dao Nguyen, Amruta Joshi-Datar, Francois Lepine, Elizabeth Bauerle, Oyebode Olakanmi, Karlyn Beer, Geoffrey McKay, Richard Siehnel, James Schafhauser, Yun Wang, Bradley E Britigan, and Pradeep K Singh. Active starvation responses mediate antibiotic tolerance in biofilms and nutrient-limited bacteria. Science, 334(6058):982-986, November 2011.

[12] Vasili Hauryliuk, Gemma C Atkinson, Katsuhiko S Murakami, Tanel Tenson, and Kenn Gerdes. Recent functional insights into the role of (p)ppgpp in bacterial physiology. Nat. Rev. Microbiol., 13(5):298-309, May 2015.

[13] Regine Hengge-Aronis. Signal transduction and regulatory mechanisms involved in control of the $\sigma \mathrm{s}$ (rpos) subunit of rna polymerase. Microbiology and molecular biology reviews, 66(3):373-395, 2002.

[14] Rv Lange and Regine Hengge-Aronis. Identification of a central regulator of stationary-phase gene expression in escherichia coli. Molecular microbiology, 5(1):49-59, 1991.

[15] Rebecca Page and Wolfgang Peti. Toxin-antitoxin systems in bacterial growth arrest and persistence. Nature chemical biology, 12(4):208-214, 2016. 
[16] Sylvie Manuse, Yue Shan, Silvia J Canas-Duarte, Somenath Bakshi, Wei-Sheng Sun, Hirotada Mori, Johan Paulsson, and Kim Lewis. Bacterial persisters are a stochastically formed subpopulation of lowenergy cells. PLoS biology, 19(4):e3001194, 2021.

[17] Susan A Moore, DMe Moennich, and MJ Gresser. Synthesis and hydrolysis of adp-arsenate by beef heart submitochondrial particles. Journal of Biological Chemistry, 258(10):6266-6271, 1983.

[18] Brian P Conlon, Sarah E Rowe, Autumn Brown Gandt, Austin S Nuxoll, Niles P Donegan, Eliza A Zalis, Geremy Clair, Joshua N Adkins, Ambrose L Cheung, and Kim Lewis. Persister formation in staphylococcus aureus is associated with atp depletion. Nature microbiology, 1(5):1-7, 2016.

[19] Yingying Pu, Yingxing Li, Xin Jin, Tian Tian, Qi Ma, Ziyi Zhao, Ssu-yuan Lin, Zhanghua Chen, Binghui Li, Guang Yao, et al. Atp-dependent dynamic protein aggregation regulates bacterial dormancy depth critical for antibiotic tolerance. Molecular cell, 73(1):143-156, 2019.

[20] Avinash Patel, Liliana Malinovska, Shambaditya Saha, Jie Wang, Simon Alberti, Yamuna Krishnan, and Anthony A Hyman. Atp as a biological hydrotrope. Science, 356(6339):753-756, 2017.

[21] JJ Heijnen. Impact of thermodynamic principles in systems biology. In Biosystems engineering II, pages 139-162. Springer, 2010.

[22] Markus Basan, Tomoya Honda, Dimitris Christodoulou, Manuel Hörl, Yu-Fang Chang, Emanuele Leoncini, Avik Mukherjee, Hiroyuki Okano, Brian R Taylor, Josh M Silverman, et al. A universal trade-off between growth and lag in fluctuating environments. Nature, 584(7821):470-474, 2020.

[23] Christophe Chassagnole, Naruemol Noisommit-Rizzi, Joachim W Schmid, Klaus Mauch, and Matthias Reuss. Dynamic modeling of the central carbon metabolism of escherichia coli. Biotechnology and bioengineering, 79(1):53-73, 2002.

[24] Hiroyuki Kurata and Yurie Sugimoto. Improved kinetic model of escherichia coli central carbon metabolism in batch and continuous cultures. Journal of bioscience and bioengineering, 125(2):251$257,2018$.

[25] Tuty Asmawaty Abdul Kadir, Ahmad A Mannan, Andrzej M Kierzek, Johnjoe McFadden, and Kazuyuki Shimizu. Modeling and simulation of the main metabolism in escherichia coli and its several single-gene knockout mutants with experimental verification. Microbial cell factories, 9(1):1-21, 2010.

[26] Yukako Tohsato, Kunihiko Ikuta, Akitaka Shionoya, Yusaku Mazaki, and Masahiro Ito. Parameter optimization and sensitivity analysis for large kinetic models using a real-coded genetic algorithm. Gene, 518(1):84-90, 2013.

[27] Oliver Kotte, Judith B Zaugg, and Matthias Heinemann. Bacterial adaptation through distributed sensing of metabolic fluxes. Molecular systems biology, 6(1):355, 2010.

[28] Kirill Peskov, Ekaterina Mogilevskaya, and Oleg Demin. Kinetic modelling of central carbon metabolism in escherichia coli. The FEBS journal, 279(18):3374-3385, 2012.

[29] Ahmad A Mannan, Yoshihiro Toya, Kazuyuki Shimizu, Johnjoe McFadden, Andrzej M Kierzek, and Andrea Rocco. Integrating kinetic model of e. coli with genome scale metabolic fluxes overcomes its open system problem and reveals bistability in central metabolism. PloS one, 10(10):e0139507, 2015.

[30] Jimena Di Maggio, JC Diaz Ricci, and M Soledad Diaz. Global sensitivity analysis in dynamic metabolic networks. Computers \& Chemical Engineering, 34(5):770-781, 2010.

[31] Katja Bettenbrock, Sophia Fischer, Andreas Kremling, Knut Jahreis, Thomas Sauter, and Ernst-Dieter Gilles. A quantitative approach to catabolite repression in escherichia coli. Journal of Biological Chemistry, 281(5):2578-2584, 2006. 
[32] Vivek Kumar Singh and Indira Ghosh. Kinetic modeling of tricarboxylic acid cycle and glyoxylate bypass in mycobacterium tuberculosis, and its application to assessment of drug targets. Theoretical Biology and Medical Modelling, 3(1):1-11, 2006.

[33] C Juan TORRES, Victoria Guixé, and Jorge Babul. A mutant phosphofructokinase produces a futile cycle during gluconeogenesis in escherichia coli. Biochemical Journal, 327(3):675-684, 1997.

[34] Yikun Tan and James C Liao. Metabolic ensemble modeling for strain engineers. Biotechnology journal, $7(3): 343-353,2012$.

[35] Linh M Tran, Matthew L Rizk, and James C Liao. Ensemble modeling of metabolic networks. Biophysical journal, 95(12):5606-5617, 2008.

[36] Ali Khodayari, Ali R Zomorrodi, James C Liao, and Costas D Maranas. A kinetic model of escherichia coli core metabolism satisfying multiple sets of mutant flux data. Metabolic engineering, 25:50-62, 2014.

[37] Ali Khodayari and Costas D Maranas. A genome-scale escherichia coli kinetic metabolic model k-ecoli457 satisfying flux data for multiple mutant strains. Nature communications, 7(1):1-12, 2016.

[38] Matthew L Rizk and James C Liao. Ensemble modeling for aromatic production in escherichia coli. PloS one, 4(9):e6903, 2009.

[39] Yue Shan, Autumn Brown Gandt, Sarah E Rowe, Julia P Deisinger, Brian P Conlon, and Kim Lewis. Atp-dependent persister formation in escherichia coli. MBio, 8(1), 2017.

[40] Palsson B Orth J, Fleming R. Reconstruction and Use of Microbial Metabolic Networks: the Core Escherichia coli Metabolic Model as an Educational Guide. 2010.

[41] Zachary A King, Andreas Dräger, Ali Ebrahim, Nikolaus Sonnenschein, Nathan E Lewis, and Bernhard O Palsson. Escher: a web application for building, sharing, and embedding data-rich visualizations of biological pathways. PLoS Comput Biol, 11(8):e1004321, 2015.

[42] Athel Cornish-Bowden. Fundamentals of enzyme kinetics. John Wiley \& Sons, 2013.

[43] R Patnaik, WD Roof, RF Young, and JC Liao. Stimulation of glucose catabolism in escherichia coli by a potential futile cycle. Journal of bacteriology, 174(23):7527-7532, 1992.

[44] Oliver Hädicke, Katja Bettenbrock, and Steffen Klamt. Enforced atp futile cycling increases specific productivity and yield of anaerobic lactate production in escherichia coli. Biotechnology and bioengineering, 112(10):2195-2199, 2015.

[45] Minoru Kanehisa and Susumu Goto. Kegg: kyoto encyclopedia of genes and genomes. Nucleic acids research, 28(1):27-30, 2000.

[46] F. Pedregosa, G. Varoquaux, A. Gramfort, V. Michel, B. Thirion, O. Grisel, M. Blondel, P. Prettenhofer, R. Weiss, V. Dubourg, J. Vanderplas, A. Passos, D. Cournapeau, M. Brucher, M. Perrot, and E. Duchesnay. Scikit-learn: Machine learning in Python. Journal of Machine Learning Research, 12:2825-2830, 2011. 


\section{Supplementary Files}

This is a list of supplementary files associated with this preprint. Click to download.

- SIDataset.zip

- SIText.pdf 